\title{
Therapeutic Effect of Hyaluronic Acid on Experimental Osteoarthrosis of Ovine Temporomandibular Joint
}

\author{
Chang-Hwan KIM ${ }^{1)}$, Beom-Jun LEE ${ }^{2)}$, Junghee YOON ${ }^{3)}$, Kang-Moon SEO ${ }^{4)}$, Jong-Hwan PARK ${ }^{1)}$, Jin-Won LEE ${ }^{1)}$, \\ Eun-Sil $\mathrm{CHOI}^{1)}$, Jung-Ju HONG ${ }^{1)}$, Yong-Soon $\mathrm{LEE}^{2)}$ and Jae-Hak PARK ${ }^{1) *}$ \\ ${ }^{1)}$ Departments of Laboratory Animal Medicine, ${ }^{2)}$ Veterinary Public Health and ${ }^{3)}$ Veterinary Radiology, College of Veterinary Medicine, \\ Seoul National University, Suwon 441-744, Korea and ${ }^{4)}$ Department of Animal Surgery, College of Veterinary Medicine, Kangwon \\ National University, Chunchon, Korea
}

(Received 26 December 2000/Accepted 25 June 2001)

ABSTRACT. A symptomatic relief by hyaluronic acid (HA, MW: $3.5 \times 10^{6}$ ), which is synthesized by Streptococcus spp, was investigated in experimental ovine osteoarthrosis. Bilateral osteoarthrosis (OA) of the temporo-mandibular joints (TMJs) was induced by perforating discs and by scrapping subchondral condylar surface. HA was intra-articularly injected into the left joints of 6 sheep on 7, 10, 14, 17 and 21 days after the operation and physiological saline as the control was injected into the contralateral (right) joints on the same day. Three sheep were killed at 1 month post-operation (MPO) and the remaining three sheep were killed at 3 MPO. Various responses such as proliferation of fibrous tissue, denudation, erosion, osteophyte formation, subcortical cyst formation and ankylosis were observed radiographically and histopathologically. The treatment of HA ameliorated the degenerative changes and lowered the osteoarthrotic score in the left joints at 1 MPO (9.96 vs 5.81) and 3 MPO (10.86 vs 5.29) compared to the right joints. These results indicate that a repeated intra-articular injection of HA inhibits the progression of OA in ovine TMJs by inducing the development of articular cartilage and by reducing the proliferation of fibrotic tissue.

KEY WORDS: hyaluronic acid, osteoarthrosis, ovine, temporomandibular joint.

J. Vet. Med. Sci. 63(10): 1083-1089, 2001

Osteoarthritis (OA) is a chronic non-inflammatory degenerative disease of synovial joint. It is a heterogeneous condition that causes pathogenic changes that are presumably irreversible. The characteristic pathology includes fibrillation of the joint surface, eburnation of articular cartilage, synovitis, and subchondral bone changes [7]. In many cases, knee pain is often progressive and leads the patient to seek medical attention [14]. Pharmacologic and nonmedical treatments are, in most cases, only modestly successful in relieving pain. It appears that naturally derived hyaluronic acid (HA) and newer formulations available on the market belong to the pharmacological class of slow-acting drugs for the treatment of OA. These compounds seem to have the potential to modulate the painful symptoms of OA as well as to improve the function of the OA joint $[11,26]$.

HA, which is a linear and unbranched polysaccharide consisting of repeated disaccharide units, functions as the backbone of the proteoglycan aggregates necessary for the functional integrity of articular cartilage of the knee [25]. The aggregate is surrounded by collagen mesh fiber to form the main content of cartilage matrix [25]. Under the pathologic conditions such as OA, rheumatoid arthritis and infectious arthritis, the concentrations and molecular weight of HA in the joint are reduced, thereby resulting in the change of the nature of the synovial fluid [5,8].

Few in vivo data support that HA formulations could have a structure-modifying effect on human osteoarthritis carti-

\footnotetext{
* Correspondence to: Park, J.-H., Department of Laboratory Animal Medicine, College of Veterinary Medicine, Seoul National University, 103 Seodun-dong, Kwonsun-gu, Suwon 441-744, Korea.
}

lage $[11,14]$. Animal-based studies have demonstrated positive effects of exogenous HA on pain in the joint, heat shock proteins, and in models of OA [3, 9, 16, 20-22, 24]. The positive clinical and experimental consequences are based on direct and indirect effects of viscosupplementation associated with a normalization of the rheologic properties of the osteoarthritic synovial fluid, decreased inflammation, and end-coating of the pain receptors in the osteoarthritic joint.

Several companies have developed HAs for the treatment of OA and the molecular weights of the HAs were smaller than natural HA present in normal synovial fluid [1]. In the present study, we have isolated HA (MW: $3.5 \times 10^{6}$ ) produced by Streptococcus spp. Its characteristics are very similar to those of HA synthesized in humans. The therapeutic effect of HA on experimentally induced osteoarthrosis in temporomandibular joint of sheep was investigated in an ovine animal model.

\section{MATERIALS AND METHODS}

Animals: Ten sheep weighing approximately $60 \mathrm{~kg}$ were purchased from Haesung Farm (Suwon, Korea) and reared at the sheep farm at Seoul National University. The animals were grown in field condition. Food and water were fed $a d$ libidum.

Hyaluronic acid: Sodium hyaluronate was obtained from LG Chemical Co. Ltd. (Seoul, Korea). The HA was isolated from HA capsule of Streptococcus spp. and the molecular weight of the HA was $3.5 \times 10^{6}$. HA was stored at $4^{\circ} \mathrm{C}$ until use. 
Induction of $O A$ : Six animals were anesthetized with an intramuscular injection of $4 \mathrm{~m} l$ of $2 \%$ xylazine hydrochloride. The preauricular region was shaved and draped in a sterile manner. After the animals were restrained, preauricular skin incision was performed to approach to the joint capsule. The sheep were subjected to disc perforation (5 $\mathrm{mm}$ in diameter) by biopsy puncture and to removal of the condylar surface by curette in the TMJ bilaterally. Antibiotics (a mixture of penicillin and streptomycin) were applied for 1 week.

Injection of the HA: HA was dissolved in physiologic saline to create $1 \%$ solution ( $1 \mathrm{~m} l$ per syringe). At $7,10,14$, 17 , and 21 days after the operation, $1 \mathrm{ml}$ of HA was injected into the left superior joint surface and $1 \mathrm{ml}$ of physiologic saline was injected into the right superior joint surface. After completion of the intra-articular injections, the sheep were returned to the field condition. Three sheep were killed by bleeding from cervical artery after an anesthetic overdose $(0.8 \mathrm{ml}$ of $2 \%$ xylazine hydrochloride $)$ at 1 month post-operation (MPO) and the remaining three sheep at 3 MPO. Two sheep were not operated but treated with $1 \mathrm{ml}$ of saline and two sheep were not operated and treated either. The joint areas were removed en bloc with a band saw and fixed in $10 \%$ neutral buffered formalin.

Radiological examination: The joint blocks were radiographically examined in the anteroposterior and lateral planes under standardized conditions by the method described by Ogi et al. [17]. The following features on osteoarthrosis were assessed for both the condylar and temporal surfaces: erosion, flattening, osteophyte formation, sclerosis, and presence of subcortical cysts. For evaluation of each radiological feature, a rating of 0 for no demonstrable change, 1 for mild (change in a third of joint surface), 2 for moderate (change in two-thirds of joint surface), and 3 for severe change (whole joint surface) was used. This was applied to both radiographic planes, all the radiological features, and both joint surfaces: therefore, a maximum score of 60 could be assigned to a joint.

Histopathological examination: The joint areas were decalcified with $9.5 \%$ hydrochloric acid, $1 \%$ sodium acetate, and saturated EDTA. The decalcified joints were cut in the parasagittal plane into lateral, central and medial sections. The samples were processed in graded alcohol and embedded in paraffin. The blocks were sectioned with 3 $\mu \mathrm{m}$-thickness and stained with hematoxylin and eosin. The slides were assessed at their anterior, central and posterior aspects; with lateral, central and medial specimens, a joint was, thus, divided into nine zones. As shown in Table 1, each zone was histopathologically assessed for the osteoarthrotic stage by a modified classification of the method described by Yoshimi et al. [28] and Mankin et al. [15].

Statistical analysis: The results were analyzed using a Wincon 2-sample test and a Kruskal-Wallis test at $\mathrm{p}<0.05$.

\section{RESULTS}

Radiography: Osteoarthrosis was induced by disk perfo-
Table 1. Osteoarthritic score for histopathological findings in TMJ of sheep

\begin{tabular}{cl}
\hline Score & \multicolumn{1}{c}{ Lesions } \\
\hline 0 & No change \\
1 & Fibrillation, mild \\
2 & Fibrillation, severe \\
3 & Cleft in fibrocartilage of the condyle \\
4 & Cleft in calcified cartilage \\
5 & Denudation, eburnation \\
6 & Erosion of bone, flattening, subcortical cyst \\
7 & Fibrosis of bone marrow \\
8 & Granulation, fibrous repair \\
9 & Fibrous ankylosis \\
\hline
\end{tabular}

ration and by bilateral removal of condylar cartilage in the joints of sheep. In saline-treated joints, radiographic abnormalities was not seen in the temporal bone, whereas a marked osteophyte formation was seen in the condyle at 1 MPO, with being greater at 3 MPO (Figs. 1a \& 2a). Erosion and flattening were also seen in the condyles of the salinetreated osteoarthrotic joints at 3 MPO (Fig. 2a). The injection of HA into the experimental osteoathrotic lesion ameliorated the morphological changes seen in the salinetreated contralateral right joints. In HA-treated joints, the osteophyte formation was also seen in the condyles, but the degree of the change was much less severe than that in the condyles of the contralateral joints (Figs. 1b \& 2b). The average radiologic rating of the HA-treated joints was 15.3 with a range of $12-18$, whereas that of the contralateral joints was 25.3 with a range of 23-27.

Osteoathrotic score: The degree of osteoarthrosis was evaluated by a scoring system (Table 1). The average osteoarthrotic scores in the HA-treated joints were statistically valuable at 1 MPO $(p<0.01)$ and 3 MPO $(p<0.01)$, compared to those in the saline-treated contralateral joints. The osteoarthrotic score in the centro-lateral, anterio-central, centro-central, anterio-medial, and centro-medial parts of the TMJ were significantly $(p<0.01)$ different from those of the appropriate controls at 1 MPO (Table 2). The osteoarthrotic score in the centro-lateral, posterio-lateral, anteriocentral, centro-central, posterio-central, anterio-medial, and centro-medial parts of the TMJ were significantly $(\mathrm{p}<0.01)$ different from those of the appropriate controls at $3 \mathrm{MPO}$ (Table 2).

Histopathological findings: Normal sheep articular stratification of mandibular condyle. Articular fibrous layer, proliferative zone, fibrocartilage zone, calcified cartilage zone were seen downward (Fig. 3) .

Histopathologically, various pathological changes including denudation, erosion, and loss of normal multiple layer structure in the condyle were observed in the salinetreated osteoarthrotic joints at 1 MPO (Fig. 4a). The superficial fibrous layer of condyles was thickened due to proliferation of fibrous tissue and blood vessels (Fig. 4a). The boundary between fibrous layer and cartilage layer was unclear. The fibrous layer was invaded to bone marrow. 

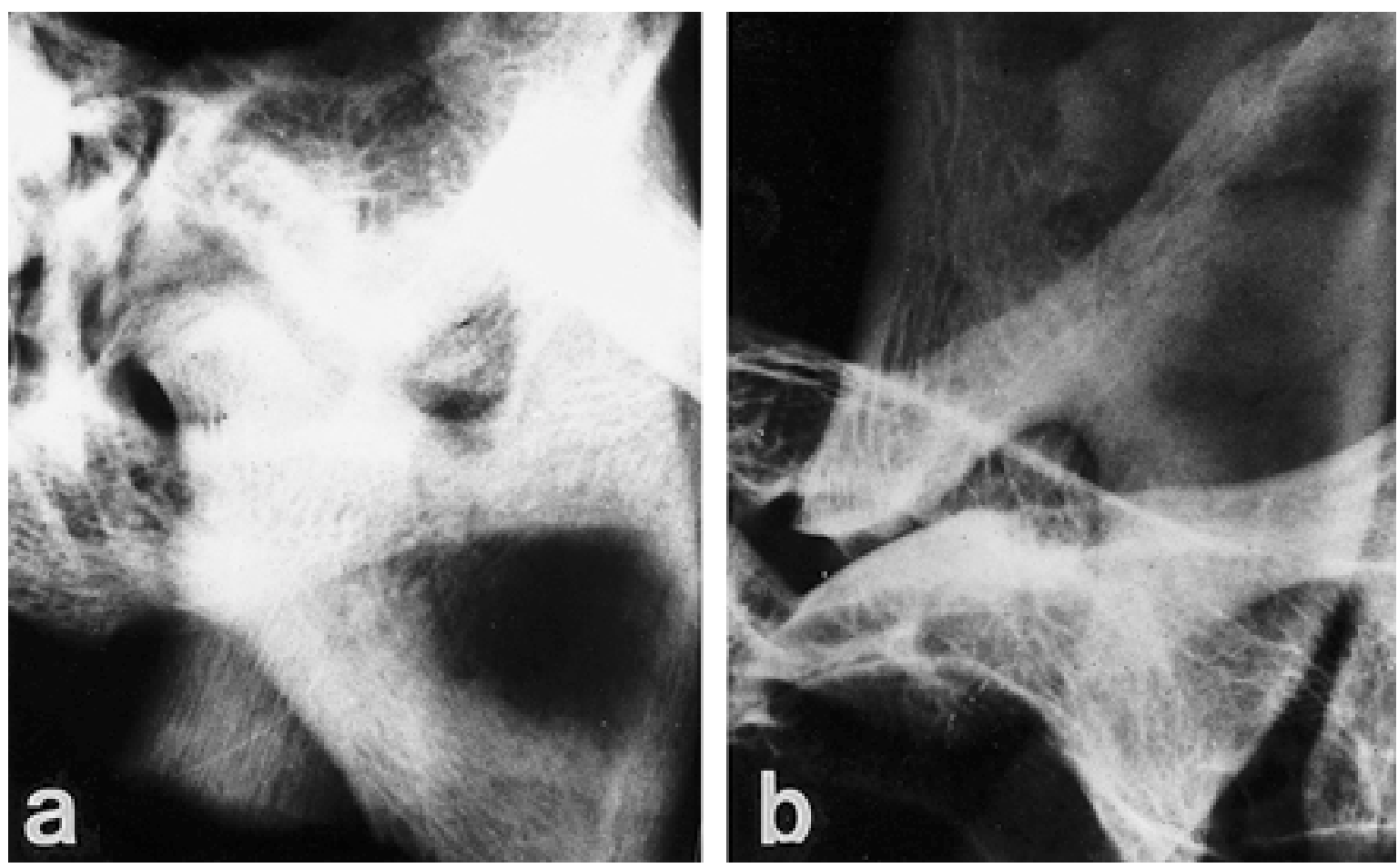

Fig. 1. Lateral plane radiograph of TMJ treated with (a) physiological saline or (b) hyaluronic acid at 1 month post-operation in an ovine experimental arthrotic model. Condylar articular surface proliferated toward temporal surface with irregularities in the saline-treated joints, but temporal articular surfaces were smooth in the HA-treated joint.
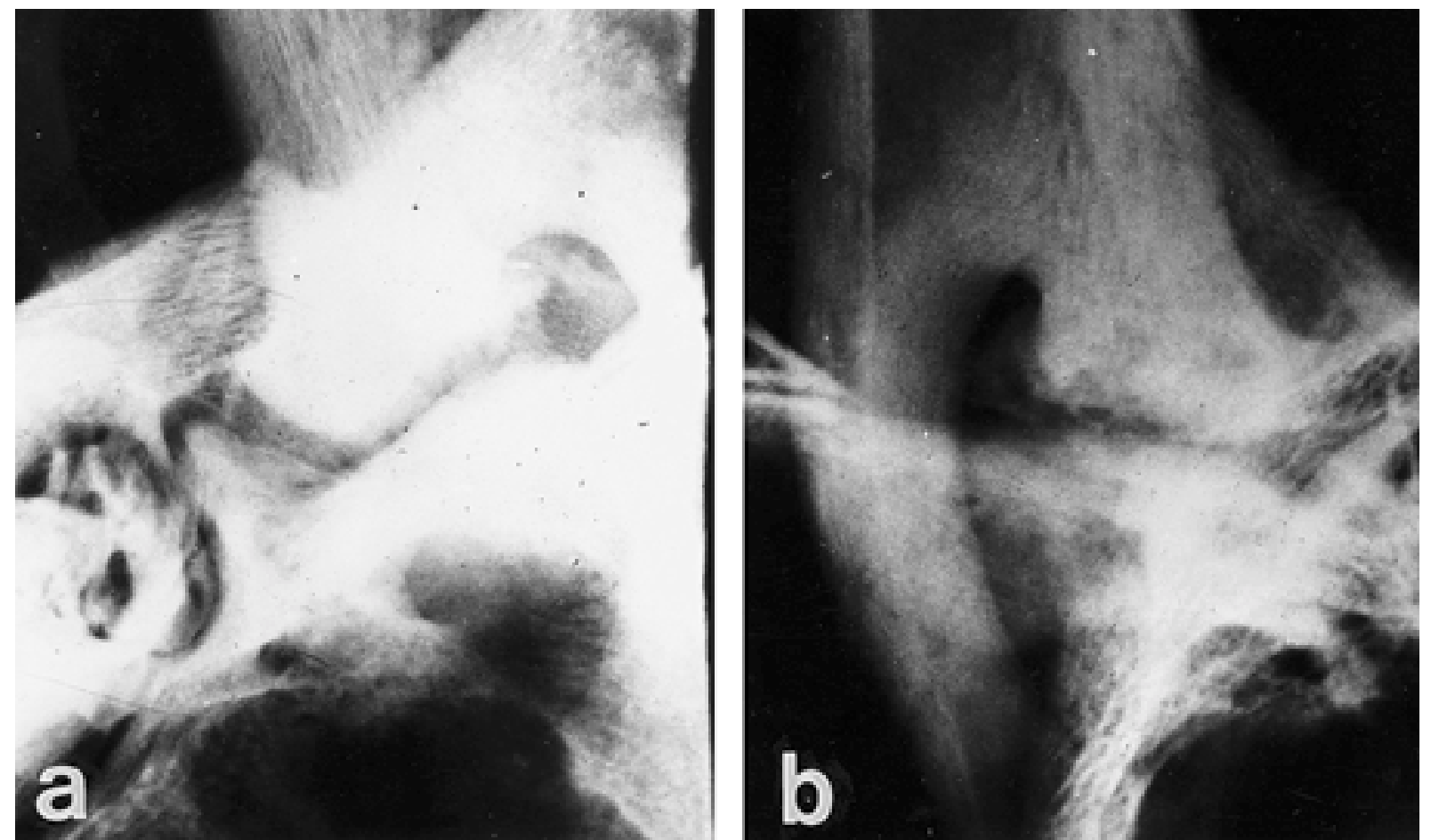

Fig. 2. Lateral plane radiograph of TMJ treated with (a) physiological saline or (b) hyaluronic acid at 3 months post-operation in an ovine experimental arthrotic model. The condylar articular surface moderately proliferated toward temporal surface with irregularity with osteophytes formation in the saline-treated joints, whereas condylar articular surface showed flattening and mild irregularities in the HA-treated joints. 
Table 2. Osteoarthrotic score of temporal mandibular joint in sheep

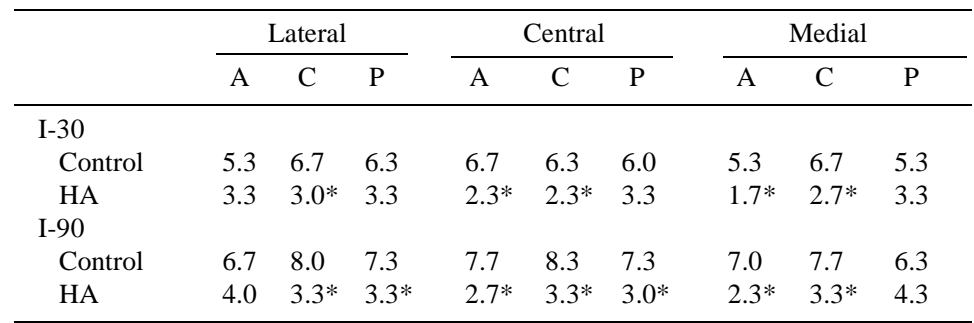

A: anterior; C: central; P: posterior. Data represent the mean of three sheep.

$*$ Significantly different from appropriate control $(\mathrm{p}<0.01$, Wincon 2-Sample Test and Kruskal-Wallis Test).

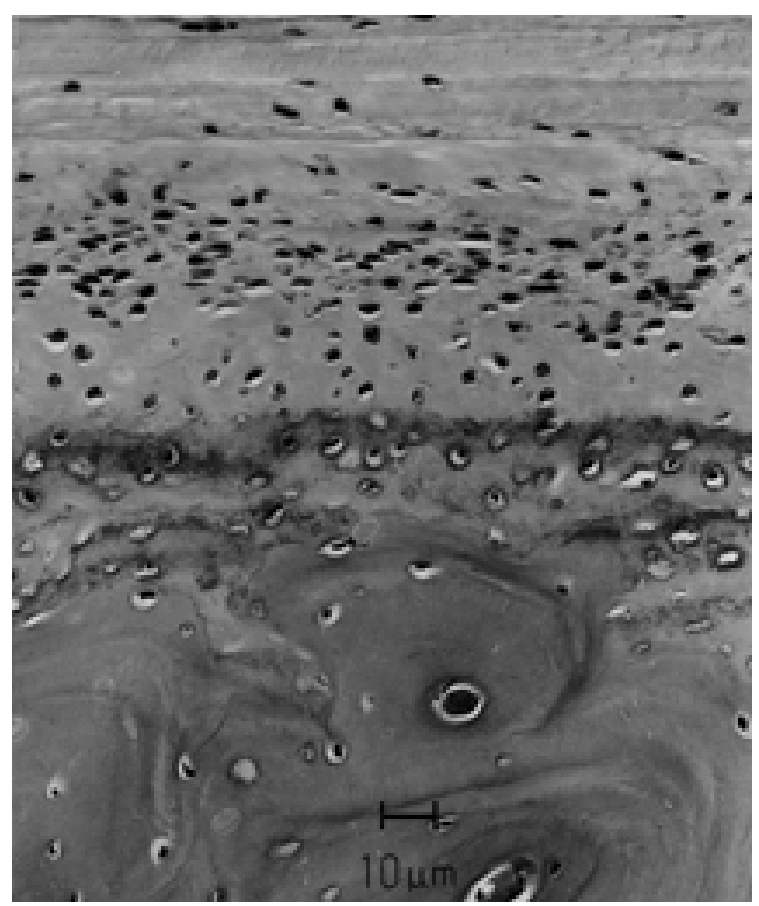

Fig. 3. Normal sheep articular stratification of mandibular condyle. Articular fibrous layer, proliferative zone, fibrocartilage zone, calcified cartilage zone were seen downward.

Proliferated collagen fibers were increased as a mass perpendicular to the articular surface (Fig. 4a). In bone tissue of the condyle, flattening of extreme part, osteophyte formation, and subcortical cyst were observed. Bone marrow fibrosis was also seen in the articular surface and denuded bone. These changes were much progressive and severe at 3 MPO. Proliferation of fibrous tissue was widespread through articular surface and marked bone marrow fibrosis was observed (Fig. 5a).

The treatment of HA tempered and mitigated the pathological features seen in the saline-treated osteoathrotic joints. Fibrocartilage proliferation was seen in the bone surface removed by curette (Fig. 4b). Fibrillation and/or cleft of the articular surface were randomly extended to the calci- fied cartilage (Fig. 4b). Bone marrow fibrosis was lessened. Blood vessels, which were observed in the proliferated fibrous tissue in the saline-treated joints, were not observed in the HA-treated joints (Fig. 4b). The cartilage layer were thickened and many regenerated chondrocytes were seen in the proliferative zone at 3 MPO (Fig. 5b).

\section{DISCUSSION}

HA is biocompatible and is permeable to metabolites and macromolecules, so it can be used for viscosupplementation of osteoarthritis to restore proper homeostasis to the diseased fluid [4]. There are several hyaluronan products approved for intra-articular use in humans [1]. They vary in the average molecular weight from a low of about $0.65 \times 10^{6}$ to about $2 \times 10^{6}$. It is important to note that the HA in normal synovial fluid has a molecular weight of about $2-3 \times$ $10^{6}$, and that HA in osteoarthritic synovial fluid has a molecular weight of about $6 \times 10^{5}$. Until now, the molecular weight of the HA products that have been marketed is not higher than that in normal synovial fluid, nor do these products have a long residence time in the joint. Because HA is a linear polysaccharide, its molecular weight is significantly decreased by only a few breaks in the molecule, and HA is very susceptible to free radical degeneration [18].

In animal studies, the molecular weight of the HA in the synovial fluid is higher, longer after the injected HA is gone [4]. Furthermore, the injection of a high-molecular weight HA was much better for the treatment of osteoarthritis than a low-molecular weight HA [13]. Though there had been, until recently, no direct comparison of HA products of differing molecular weight in humans, a consideration of the results from all these studies suggests that the efficacy is improved with a higher molecular weight product [19]. We have developed a new HA drug that is synthesized by Streptoccocus spp. and of which molecular weight is $3.5 \times 10^{6}$. In this study, a repeated injection of the HA minimized the extent of the osteoarthrotic changes induced operatively in TMJ of sheep. The control joint showed similar changes to those previously shown in the OA model [12]. The model used was a combination of two previously published techniques $[6,12]$. The disc perforation was added to the condylar scarification to ensure that the circulation of the HA 


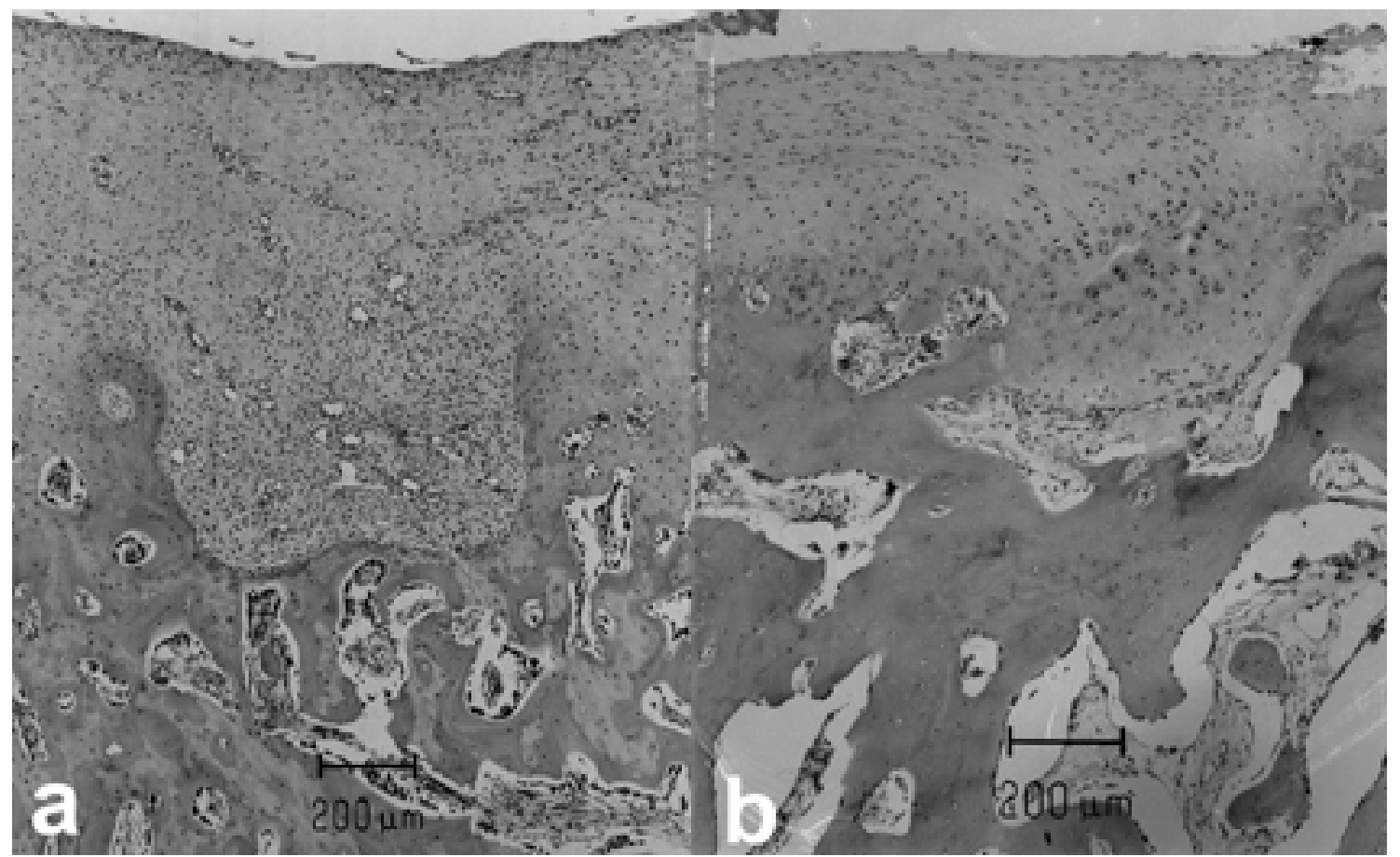

Fig. 4. Sheep TMJ (central posterior) treated with (a) phosphate buffered saline or (b) HA at 30 days post operation. Fibrosis of bone marrow and granulation tissue on denuded bone and articular surface denudation were seen in the condylar bone of control sheep. A mild fibrosis in the bone marrow was seen and the regenerated chondrocytes were seen increased on the denuded condylar bone in the HA-treated sheep .

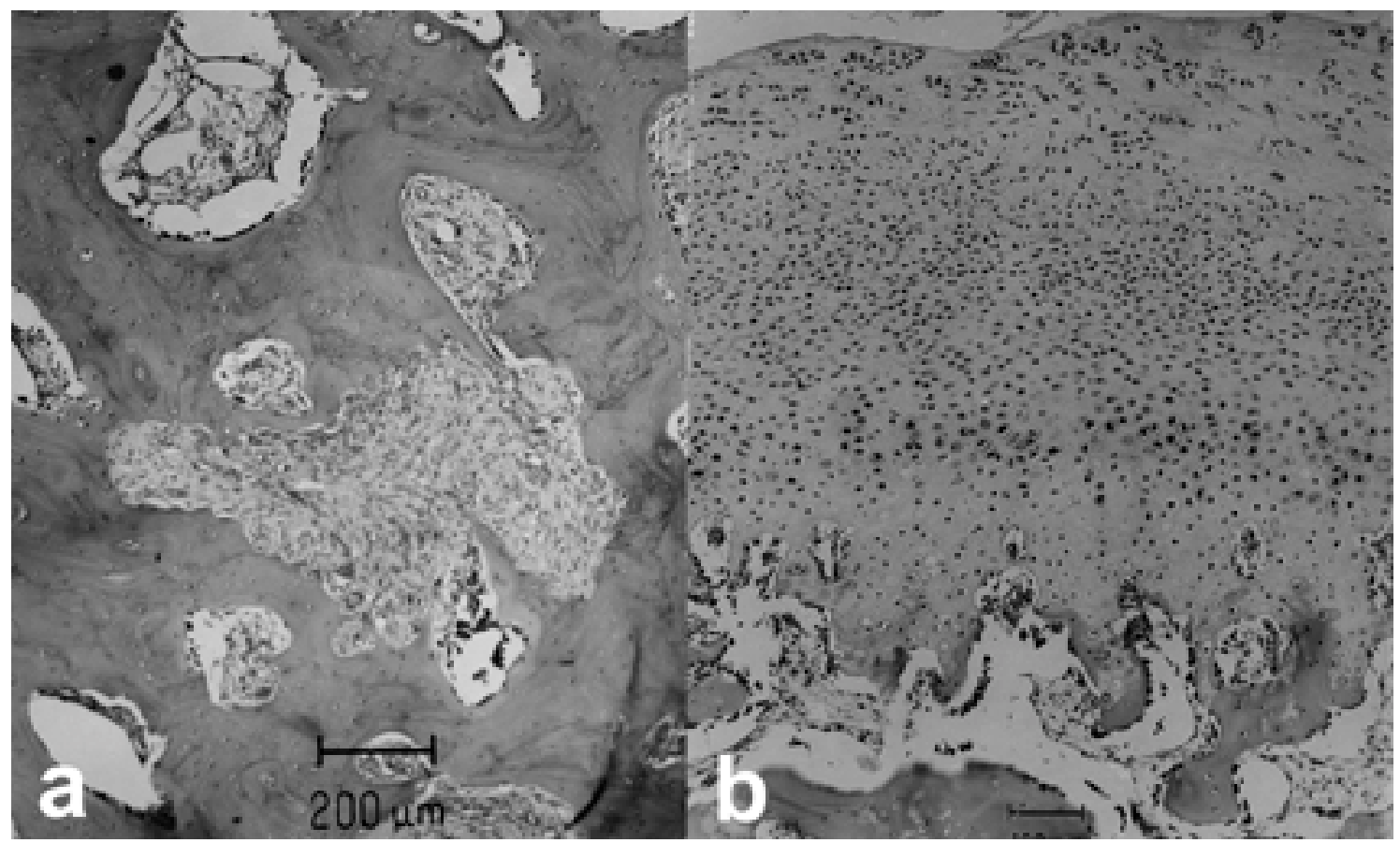

Fig. 5. Sheep TMJ (lateral anterior) treated with (a) phosphate buffered saline or (b) HA at 90 days post operation. Fibrosis of bone marrow were severely occurred in the TMJ of control sheep. Articular cartilage was proliferated in the HA-treated TMJ. 
would be throughout the joint space after a single injection into the superior joint space. Thus its effect on the superficial condylar scarification model could be checked, knowing that HA would directly affect the site of damage. The result for the control side was the same as that for the condylar scarification OA model, the added disc perforation having no observable effect. This is not surprising because a perforation model alone resulted in minimal intra-articular changes, mainly hyperplasia of the articular surface in the vicinity of the perforation. Most of the condyles in the scraping OA model developed a disc perforation within the first month, so the artificial disc perforation was only a variable in early phases [6].

The promising effect of HA in this study may be either mechanical or metabolic. The mechanical concept is simple, namely, that the HA maintains lubrication and thus minimizes wear and tear. In fact, previous studies have reported decreased HA concentration in synovial fluid in pathologic joints. Therefore, decreased HA may result in increased friction in the intraarticular space, and intraarticularly injected HA may contribute to the reduction of this friction. However, the injections were spaced 3 or 4 days apart, and the half-life of HA within a joint is much less than that, being reported at approximately $13 \mathrm{hr}$ [2]. Therefore, the mechanical effect of injected HA may only be effective during that early period.

Metabolically, HA has a role in nutrition of avascular parts of the disc and the condylar cartilage [10, 27]. It also has a structural role in cartilage formation in which glycosaminoglycans are bound to HA by link-proteins to form the matured articular proteoglycan [27]. In pathological conditions, proteoglycans of the cartilage may be disaggregated and dispersed into the synovial cavity. Thus, the addition of the HA may act to protect the cartilage from further degradation by coating the damaged surface.

Recent studies have shown that many proteolytic enzymes and cytokines can be identified in the OA process. Cytokines such as interlukin-1,6 and tumor necrosis factor levels become increased, which enhances the matrix metalloproteinases and as a result the matrix becomes damaged [23]. Intraarticular injection of HA may decrease these inflammatory mediators. Another possibility is that HA injection stimulates increased natural HA production by the synovial cells [4]. Recent studies have suggested that HA has a potential to form complexes with phospholipids, including platelet-activating factor and metabolites such as prostagladins [9]. Thus, injection of highly purified HA may also have some capacity to form complexes with phospholipids and metabolites, thereby reducing the inflammatory mediators in the synovial cavity that could contribute to the inhibition of cartilage matrix degradation. Although this mechanism of HA activity is still controversial, the results of this study indicate that HA (MW: $3.5 \times 10^{6}$ ) can be used as a prominent therapeutic agent for osteoarthrosis.

ACKNOWLEGMENT. The BK 21 of the Ministry of Education supported this study.

\section{REFERENCES}

1. Adams, M. E. 1998. Viscosupplementation as articular therapy. pp. 243-253. In: The Chemistry, Biology and Medical Applications of Hyaluronan and Its Derivatives (Laurent, T. C. ed.). Portland Press, London.

2. Antonas, K. N., Fraser, J. R. E. and Muirden, K. D. 1973. Distribution of bological labelled radioactive hyaluronic acid injection into joint. Ann. Rheum. Dis. 32: 103-111.

3. Armstrong, S., Read, E. and Ghosh, P. 1994. The effects of intraarticular hyaluronan on cartilage and subchondral bone changes in an ovine model of early osteoarthritis. J. Rheumatol. 21: 680-688.

4. Balaza, E. A and Denlinger, J. L. 1993. Viscosupplementation: a new concept in the treatment of osteoarthritis. J. Rheumatol. 20: 3-9.

5. Balazs, E. A., Watson, D., Duff, I. F. and Roseman, S. 1967. Hyaluronic acid in synovial fluid. I. Molecular parameters of hyaluronic acid in normal and arthritic human fluids. Arthritis Rheum. 10: 357-376.

6. Bosanque, A., Ishimaru, J. and Goss, A. N. 1991. Effect of experimental disc perforation in sheep temporomandibular joints. J. Oral Maxillofac. Surg. 20: 177-181.

7. Bullough, P. G. 1992. Atlas of Orthoapedic Pathology, with Clinical and Radiologic Corrections. 2nd ed., Gower Medical, New York.

8. Dahl, L.B., Dahl, I. M., Engstrom-Saurent, A. and Granath, K. 1985. Concentration and molecular weight of sodium hyaluronate in synovial fluid from patients with rheumatoid arthritis and other arthropathies. Ann. Rheum. Dis. 44: 817-822.

9. Ghosh, P., Holbert ,C., Read, R. and Armstrong, S. 1995. Hyaluronic acid (hyaluronan) in experimental osteoarthritis. $J$. Rheumatol. (Suppl.) 43: 155-157.

10. Hascall, V. C., Fulop, C., Salustri, A., Goodstone, N., Calabro, A., Hogg, M., Tammi, R., Tammi, M. and MacCallum, D. 1998. Metabolism of hyluronan. pp. 67-76. In: The Chemistry, Biology and Medical Applications of Hyaluronan and Its Derivatives (Laurent. T. C. ed.), Portland Press, London.

11. Huskisson, E. C. and Donnelly, S. 1999. Haluronic acid in the treatment of osteoarthritis of the knee. Rheumatology 38: 602607.

12. Ishimaru, J. and Goss, A. N. 1992. A model for osteoarthritis of the temporomandibular joint. J. Oral Maxillofac. Surg. 50: 1191-1195.

13. Kikuchi, T., Yamada, H. and Shinmei, M. 1996. Effect of high molecular weight hyaluroan on cartilage degeneration in a rabbit model of osteoarthritis. Osteoarthritis Cartilage 4: 99-110.

14. Lesile, M. 1999. Hyaluronic acid treatment for osteoarthritis of the knee. Nurse Pract. 24: 41-48.

15. Mankin, H. J., Dorfman, H., Lippiello, L. and Zarins, A. 1971. Biochemical and metabolic abnormalities in articular cartilage from osteoarthritic human hips. J. Bone Joint Surg. Am. 53A: 523-537.

16. Neo, H., Ishimaru, J., Kurita, K. and Goss, A. N. 1997. The effect of hyaluronic acid on experimental temporomandibular joint osteoarthrosis in the sheep. J. Oral Maxillofac. Surg. 55: 1114-1119.

17. Ogi, N., Kurita, K.,.Handa, Y. and Goss, A. N. 1996. The short-term effect of discectomy on the osteoarthrotic temporomandibular joint in sheep. J. Oral Maxillofac. Surg. 25: 319 324.

18. Phillips, G. O. 1998. Degradation of hyluronan systems by free radicals. pp. 93-112. In: The Chemistry, Biology and Medical 
Applications of Hyaluronan and Its Derivatives (Laurent, T. C. ed.), Portland Press, London.

19. Phillips, M. 1989. Clinical trial comparison of intra-articular sodium hyaluronic acid products in the horse. Equine Vet. Sci. 9: $39-40$.

20. Rydell, N. and Balazs, E. A. 1971. Effect of intraarticular injection of hyaluronic acid on the clinical symptoms of osteoarthritis and on granulation tissue formation. Clin. Orthopedics Rel. Res. 80: 25-32.

21. Sakakibara, Y., Miura, T., Iwata, H., Kikuchi, T., Yamaguchi, T., Yoshimi, T. and Itoh, H. 1994. Effect of high-molecularweight sodium hyaluronate on immobilized rabbit knee. Clin. Orthopedics Rel. Res. 299: 282-292.

22. Shimazu, C., Yoshioka, M., Coutts, R. D., Harwood, F. L., Kubo, T., Hirasawa, Y. and Amiel, D. 1998. Long-term effects of hyaluronan on experimental osteoarthritis in the rabbit knee. Osteoarthritis Cartilage 6: 1-9.

23. Shinmei, M., Okada, Y., Masuda, K., Naramatsu, M., Kikuchi, T., Harigawa, M. and Shimomura, Y. 1990. The mechanism of cartilage degradation in osteoarthritic joint. Semin. Arthritis
Rheum. 19: 16-21.

24. Smith, G. N. Jr., Myers, S. L., Brandt, K. D. and Mickler, E. A. 1998. Effect of intraarticular hyaluronic injection in experimental canine osteoarthritis. Arthritis Rheum. 41: 976-985.

25. Toyoshima, H. 1978. The influence of synovectomy on articular cartilage of rabbit knee and preventive effects of hyaluronic acid on degenerative change of the cartilage. J. Tokyo Women's Medical College 48: 890-895.

26. Uebelhart, D. and Williams, J. M. 1999. Effects of hyaluronic acid on cartilage degradation. Curr. Opin. Rheumatol. 11: 427435.

27. Weigh, T. N., Heinegard, D. K. and Hascall, V. C. 1991. Proteiglycans: Structure and function. pp. 45-78. In: Cell biology of extracellular matrix, 2nd ed. (Hay, E. D. ed.), Plenum Press, New York.

28. Yoshimi, T., Kikuchi, T., Obara, T., Yamaguchi, T., Sakakibara, Y., Itoh, H., Iwata, H. and Miura, T. 1994. Effects of high-molecular weight sodium hyaluronate on experimental osteoarthrosis induced by resection of rabbit anterior cruciate ligament. Clin. Orthopedics Rel. Res. 298: 296-304. 